\title{
Políticas públicas de educación afrocolombiana: el arte de escamotear el derecho de los pueblos
}

\author{
Políticas públicas de educaçao afrocolombiana: a arte de escamotear \\ o direito das populações
}

\section{Public policies of afrocolombian education: the art of escaping the right of peoples}

\section{Jorge Enrique García Rincón"}

Universidad del Cauca-Colombia, Centro de Memorias Etnicas, Investigador

\begin{abstract}
Resumen: El artículo se ocupa de describir los momentos en que el Estado colombiano ha legislado para los pueblos de ancestro africano, especialmente en materia de derechos educativos. También mostrará que las leyes específicas para estas comunidades de manera contradictoria no gozan de una estrategia de promoción por parte del Estado y por el contrario se han naturalizado en un doble juego de inclusión-negación, esto es, el derecho de los negros es parte de la normatividad pero no se aplica en la práctica. Se postula aquí que la inclusión de las comunidades Afro a las políticas educativas del Estado responde a una estrategia de desmovilización e intento de cierre de los procesos reivindicativos. No obstante, pese a la visión colonial y racista que marca de forma permanente el desempeño de las elites colombianas, desde el siglo XIX emergen grupos de intelectuales negros que han logrado influir de manera significativa en los procesos de reclamación de una educación enderezada a revitalizar las construcciones sociales, políticas y culturales de los afrocolombianos. La apertura a la diversidad cultural de la Constitución política de 1991 es leída aquí como estrategia del multiculturalismo de Estado. Palabras clave: Desmovilización. Política Publica de Educación Afro. Intelectualidad Afro. Comunidad Afronariñense. Educación propia. Multiculturalismo de Estado.
\end{abstract}

Resumo: 0 artigo se ocupa em descrever os momentos em que o Estado colombiano legislou para as populações de ascendência africana, principalmente no que diz respeito aos direitos educativos. Também mostrará que as leis específicas para estas comunidades, de modo contraditório, não gozam de uma estratégia de promoção por parte do Estado. Ao contrário, foram naturalizadas em um duplo jogo de inclusão-negação, isto é, o direito dos negros é parte da normativa, mas não é aplicado na prática. Postulamos aqui que a inclusão das comunidades afrodescendentes às políticas educativas

Doctor en Ciencias de la Educación por la Universidad de Narino; Especialista en Orientación Educativa y Desarrollo Humano por la Universidad de Narino; https://orcid.org/0000-0003-4682-2125; http://lattes.cnpq.br/7020960539849712.

2 El término negro, en este artículo, tiene la misma categoría que afro. En Colombia no existe consenso sobre la manera como se debe llamar a las personas pertenecientes a los pueblos de ancestro africano. La ley 70 de 1993 habla de Comunidades Negras mientras que el discurso académico e institucional se centra en Comunidades Afrocolombianas. En cualquier caso, 
do Estado corresponde a uma estratégia de desmobilização e a uma tentativa de encerramento dos processos reivindicativos. Não obstante, apesar da visão colonial e racista que marca de forma permanente o desempenho das elites colombianas, desde o século XIX emergem grupos de intelectuais negros que conseguiram influenciar de maneira significativa os processos de reivindicação de uma educação destinada a revitalizar as construções sociais, políticas e culturais dos afrocolombianos. A abertura à diversidade cultural da Constituição política de 1991 é lida aqui como estratégia do multiculturalismo de Estado.

Palavras-chave: Desmobilização. Política Pública de Educação Afro. Intelectualidade Afro. Comunidade Afronariñense. Educação própria. Multiculturalismo de Estado.

Abstract: The article deals with describing the moments in which the Colombian State has legislated for the peoples of African ancestry, especially in the matter of educational rights. It will also show that the specific laws for these communities in a contradictory manner do not enjoy a promotion strategy on the part of the State and on the contrary have been naturalized in a double game of inclusion-denial, that is, the right of blacks is part of the normativity but it does not apply in practice. It is postulated here that the inclusion of the Afro communities to the State's educational policies responds to a demobilization strategy and an attempt to close the protest processes. However, despite the colonial and racist vision that permanently marks the performance of Colombian elites, since the nineteenth century groups of black intellectuals have emerged that have significantly influenced the processes of claiming an education aimed at revitalizing the social, political and cultural constructions of Afro-Colombians. The opening to the cultural diversity of the 1991 Political Constitution is read here as a strategy of State multiculturalism.

Keywords: Demobilization. Afro Education Public Politics. Afro intelligentsia. Afronariñense community. Own education. State Multiculturalism.

Recibido en 09 de julio de 2018 Acepto en 13 de septiembre de 2018

Publicado el 19 de febrero de 2019

\section{INTRODUCCIÓN: CONSIDERACIONES GENERALES}

De acuerdo con Maya (1998), los primeros contingentes de africanos esclavizados ingresaron a lo que hoy es Colombia por el puerto de Cartagena entre 1533 y १८10. Como ocurre en todas las naciones de América, nunca se sabrá con exactitud cuántos llegaron debido a las

en mi opinión, el lenguaje oficial que nombra a estos pueblos como comunidades negras, afrocolombianas, palenqueras y raizales está respondiendo a un criterio de clasificación de origen colonial. 
complejidades de la trata trasatlántica que vinculó legalidad (asientos) y piratería (contrabando) en el comercio de seres humanos de África trasplantados a las nuevas tierras conquistadas.

Pese a que Maya (2010) pondera la presencia de Senegambia en la Nueva Granada (actual Colombia) como iniciadores de las tradiciones y culturas afrocolombianas y pese también a que esta autora propone desyorubizar y desbantuizar las narraciones sobre la diáspora africana en el país, es innegable que los Bantues llegados a este territorio desde 1580 dejan un enorme legado cultural que es muy patente en los modos de ser de los afrocolombianos. Esa es la razón por la que aún existe en Colombia una lengua de base lingüística bantú: el palenquero, hablado en San Basilio de Palenque, territorio que fue ocupado desde finales del siglo XVII por los primeros cimarrones con Benkos Biohó a la cabeza.

Como en todos los países de América, la esclavización de africanos en Colombia fue parte del naciente sistema de dominación capitalista, colonial, patriarcal, cristianocéntrico y racista (GROSFOGUEL; CASTRO-GOMÉZ, 2007) que los europeos instauraron en América y otras latitudes del planeta asumiendo en forma arrogante que su cultura es modelo para la humanidad, con lo que se crea el falso universalismo eurocéntrico.

En la clasificación racial, cuyo propósito de fondo Quijano (2000) lo ha llamado colonialidad del poder, en tanto patrón de dominación que señala una diferenciación epistémica entre europeos y no europeos, los africanos y su descendencia con todos sus marcadores de color, fueron considerados los de menor rango en la escala social. Sometidos a trabajos forzados durante todo el día, en la minería y las plantaciones agrícolas, solo contaron con escasas horas de la noche para reinventarse en lo que Olivella (1992) denomina el Muntú en el exilio. Esto es, hombres y mujeres con su sabiduría y sus prácticas de vida espirituales y cotidianas disgregadas por efectos de la trata trasatlántica generada en el violento proceso de expansión de Europa.

Cuando la ley de abolición de la esclavitud en Colombia fue promulgada en १८51, gran parte de los africanos y sus descendientes ya habían alcanzado su libertad por la vía del cimarronismo, o por la automanumisión producto de la acumulación de oro para pagarla, en uso de una estrategia que Maya (2003) llamó cimarronaje jurídico.

En la segunda mitad del siglo XIX, personajes negros como Candelario Obeso y Luis Antonio Robles, el primero en la poesía y el segundo en la acción parlamentaria advierten el advenimiento de una intelectualidad afrocolombiana que se desarrollará con mucho brillo durante el siglo XX.

En efecto, los intelectuales negros de la Diaspora africana en Colombia Diego Luis Córdoba, Rogerio Velásquez, Miguel Antonio Caicedo Mena, Amir Smith Córdoba, Jorge Artel, Manuel Zapata Olivella, Natanael Díaz, Sofonías Yacup entre otros, van creando un campo epistémico a partir de sus discursos, ensayos, poemarios y novelas que servirán de guía 
para las negociaciones que el movimiento social afrocolombiano tendrá que enfrentar con el Estado desde la década de los años noventa del siglo XX. En el campo de la educación, algunos de los intelectuales mencionados fueron pioneros del discurso de la diferencia (GARCÍ, 2016), esto es, plantearon la necesidad de una re-localización de los lugares donde se deben producir las ideas, estrategias y proyectos de educación. En concreto, desde distintos lugares de enunciación, postularon el concepto de diversidad epistémica como principio para la educación colombiana.

En este artículo me ocuparé, siguiendo el camino trazado por los intelectuales pioneros del pensamiento educativo afrocolombiano, del análisis de la normatividad vigente que reconoce, desde un enfoque diferencial, las políticas públicas de educación para las comunidades negras; dichas políticas, en mi modo de ver, fueron adoptadas en concertación con los pueblos negros, pero sin dotación de herramientas para su aplicación práctica, y es ahí donde se percibe el tufillo de racismo, en términos de negación de la existencia física e intelectual del otro. Por lo tanto, afirmo que estas políticas han funcionado, por un lado, para dar cumplimiento a la constitución nacional que obliga a garantizar una educación para el desarrollo de la identidad de los grupos étnica y racialmente diferenciados y por otro, hay que decirlo con franqueza, solo han representado una forma de escamotear el derecho de las comunidades negras del país, en una suerte de racismo legalizado que reedita la invisibilización del negro como sujeto de negociación frente al Estado.

Los normas educativas especiales de las comunidades negras de Colombia analizadas en el presente artículo son: Ley 70 de 1993 que consagra la educación en los territorios de dichas comunidades como un proyecto de reafirmación identitaria, un proceso de revitalización cultural y en mi modo de ver una apuesta por la organización política territorial. El decreto 804 de 1995 que reglamenta la etnoeducación en perspectiva estatal descrita en la ley general de educación (ley 115 de 1994), el decreto 1122 de 1998 mediante el cual se crea la cátedra de estudios afrocolombianos y se fijan criterios de aplicación. También se retoma el postulado constitucional que indica que la educación para los grupos étnicos en Colombia debe respetar y desarrollar su identidad cultural (Art. 68 CPC).

Igualmente, son objeto de análisis en este artículo los avances en la definición de la política especial de educación para las poblaciones negras del departamento de Nariño. Este proceso podría ser considerado un ejemplo para el país en la medida en que se trata de la primera región en lograr una política de educativa en perspectiva afro.

En términos metodológicos, se analiza primero de manera sucinta el conjunto de derechos colectivos de las comunidades negras de Colombia consagrados en el artículo transitorio 55 de la constitución política y la ley 70 de 1993. De dicha ley se trabaja el capítulo VI que da origen a las dos vertientes de la educación afrocolombiana: por un lado la educación propia que debe ocurrir al interior de los territorios titulados colectivamente 
(educación propia, palenquera, etnoeducación, o afroeducación) y por otro la Cátedra de estudios afrocolombianos definida en el artículo 39 de la misma ley como la difusión obligatoria del conocimiento de las prácticas culturales propias de los pueblos negros y sus aportes a la historia y a la cultura colombiana y latinoamericana. El artículo dice también que en las áreas de sociales de los diferentes niveles educativos se incluirá la cátedra de estudios afrocolombianos conforme con los currículos correspondientes. En última instancia, se analiza la experiencia de los procesos de comunidades negras de Nariño que lograron la aprobación de la política educativa especial en debate con las autoridades oficiales de la educación en ese departamento.

Antes de los años noventa pensar en una política educativa especial para las poblaciones con ancestro africano en Colombia no estaba en los planes del Estado ni en los de las comunidades. Invisibilizados y negados como sujetos históricos, los negros no representaban una comunidad que pudiera concitar la atención del Estado. La constitución política que va desde 1886 hasta 1990 no contemplaba el reconocimiento de la diversidad étnica y cultural de la nación. Es en el momento del multiculturalismo constitucional que arranca con la carta política de 1991 en donde se logra un lugar para que las poblaciones negras discutan con el Estado la necesidad de reconocimiento de sus derechos colectivos y entre ellos los referidos a la educación. Como se verá más adelante, las reclamaciones de un proyecto educativo en perspectiva propia que se vuelve muy fuerte en el movimiento social afrocolombiano de los años noventa estuvo siempre influenciado por el pensamiento diaspórico de los intelectuales negros que desarrolla un cuestionamiento exhaustivo al sistema educativo estatal durante casi todo el siglo XX.

En el análisis de la legislación educativa afrocolombiana, en términos de su aplicación práctica, se contemplan dos perspectivas teóricas: por un lado, el pensamiento diasporico (LA0-MONTES, 2007; CAICEDO ORTIZ, 2013; GARCIÁ 2016) que asume los procesos de reivindicación en términos de crítica y cuestionamiento del ordenamiento social, político y cultural del Estado que declara la inferioridad y subalternidad del negro, negándolo como sujeto de formación y ocultando sus aportes en las ciencias sociales y humanas. El pensamiento diasporico es una re-acción de los intelectuales negros que desde distintos campos del conocimiento se han pronunciado en contra del sistema colonial de larga duración. Por otra parte, el análisis de la normatividad educativa favorable a las comunidades negras en Colombia ha requerido de las herramientas del pensamiento decolonial que cuestiona también al Estado moderno por su origen colonial y porque en la racialización como patrón de poder (QUIJANO, 2000) el racismo epistémico se afianza a partir de un sistema educativo totalmente incrustado en la matriz académica occidental o, en lo que es lo mismo, las geopolíticas del conocimiento (WALSH, 2005). Traducido en tiempos contemporáneos como democracia liberal, el sistema colonial adopta el multiculturalismo como estrategia que asume la diversidad pero procurando su asimilación a la mayor brevedad posible (HALL, 2006), no solo a las reglas del 
Estado sino también a las del mercado. Dentro de la diversidad o "subalternidad", los derechos de los negros son los de menor importancia dada su ubicación en el último escaño de la pirámide social. La educación como estrategia de movilidad social de las comunidades negras solo podrá ser una posibilidad real, en el caso colombiano, por la confrontación del racismo institucional que hacen los intelectuales negros a lo largo del siglo XX en el marco de lo que hemos llamado pensamiento crítico y educativo afrocolombiano (GARCÍA, 2016).

\section{LOS DERECHOS DE LA POBLACIÓN AFROCOLOMBIANA}

Esquilmados y negados como seres pensantes, los afrocolombianos de los ríos y barrios marginales de las principales ciudades se involucran en el debate de la Constituyente de 1991. Pese a que sus propuestas no llegaron a ser parte del articulado de la Constitución política de Colombia, la inclusión de conceptos como diversidad cultural y multietnicidad de la población colombiana, que los constituyentes de diversos sectores sociales discutieron, fue suficiente para encontrar una fisura en el nuevo ordenamiento jurídico del país favorable a la población afrocolombiana. En efecto, la Constitución en el artículo Transitorio 55 contempla y esboza el derecho de los negros a la titulación colectiva de las tierras que ocupan en las riberas de los ríos, especialmente en la región del Pacifico. Los debates acerca de los alcances de este articulo origina la ley 70 de 1993. Las comunidades afrocolombianas, en esta normativa tendrán cinco derechos básicos: titulación colectiva de los territorios ocupados, protección y desarrollo de los derechos y la identidad cultural (educación), uso y protección de los recursos naturales del territorio, derecho al desarrollo propio y derecho a la participación institucional y política.

Para lograr el reconocimiento de estos derechos, los líderes del movimiento social afrocolombiano, echaron mano de la producción intelectual de los pioneros negros del siglo XX e inician fuertes alegatos con el gobierno colombiano para garantizar no solo la normatividad, sino también su aplicación. La década de los noventa se considera el escenario de negociación histórica entre los pueblos negros y el estamento nacional. Dos temas son relevantes en la discusión: el aseguramiento de los territorios colectivos y el desarrollo del capítulo VI de la ley 70 que se ocupa de sentar las bases de lo que será la educación de estos pueblos al interior en sus territorios (GARCÍA, 2011).

De esta manera, la diáspora africana en Colombia encuentra caminos para la visibilización y reconocimiento como parte de las raíces culturales del país. En términos de creación de nación es innegable el aporte afrocolombiano a las músicas nacionales, a la convivencia pacífica, a la conservación de la naturaleza y, pese al ocultamiento deliberado 
por parte de la academia colombiana y los estudios históricos y antropológicos, las inmensas contribuciones en el campo de la literatura, la política, la economía y la convivencia de los colombianos en general.

Infortunadamente, los derechos afrocolombianos consagrados en la ley 70 solo se pueden evidenciar en la titulación colectiva de las tierras (principalmente en el Pacifico) que a la fecha superan los 6 millones de hectáreas. Sin embargo, los Consejos Comunitarios como figura de autoridad territorial son estructuras organizativas débiles que no cuentan con herramientas suficientes para administrar los territorios y carecen de la capacidad de controlar las dinámicas sociales marcadas por la violencia y el despojo que ejercen los grupos armados al margen de la ley. El derecho al desarrollo propio y el usufructo de los recursos naturales incluida la minería del oro y el platino son temas que han permanecido en silencio durante dos décadas por una actitud reprochable del Estado en prolongar los tiempos para su reglamentación ofreciendo dichos recursos a la lógica del mercado y el capital financiero internacional. En últimas, los cinco derechos básicos de la población negra colombiana no tienen condiciones para su realización plena.

\section{EL DESARROLLO DE LAS POLÍTICAS PÚBLICAS DE EDUCACIÓN AFROCOLOMBIANA}

Todos los debates de los intelectuales negros durante el siglo XX en materia de educación para la población afrocolombiana, se concretan en el capítulo VI de la ley 70 de 1993. En efecto, el estado se compromete a reconocer y garantizar a las comunidades el derecho a un proceso educativo acorde con sus necesidades y aspiraciones etnoculturales y aduce que la autoridad competente adoptará las medidas necesarias para que en cada uno de los niveles educativos, los currículos se adapten a esta disposición. (art. 32). Para una mayor comprensión y claridad acerca del alcance y la dimensión de este capítulo de la ley 70 , podemos analizar el contenido del artículo 34 que textualmente dice:

La educación para las comunidades negras debe tener en cuenta el medio ambiente, el proceso productivo y toda la vida social y cultural de estas comunidades. En consecuencia, los programas curriculares aseguraran y reflejaran el respeto y el fomento de su patrimonio económico, natural, cultural y social, sus valores artísticos, sus medios de expresión y sus creencias religiosas. Los currículos deben partir de la cultura de las comunidades negras para desarrollar las diferentes actividades y destrezas en los individuos y en el grupo, necesarios para desenvolverse en su medio social. 
Se puede apreciar que la ley es estricta en la configuración del concepto de Educación propia en las comunidades negras. No se trata del concepto de etnoeducacion que la ley 115 de 1994 (ley general de educación en Colombia) establece como servicio a los grupos étnicos del país. El concepto de educación propia está presente en todo el espíritu de este capítulo hasta el punto de diferenciar entre esta educación endógena que debe construirse "casa adentro" de las comunidades (GARCÍA, 2001) y el artículo 39 que establece la creación de la Cátedra de Estudios Afrocolombianos (CEA) como un aporte a la discusión acerca de la identidad nacional. Se entiende, a partir de esta Cátedra que no es posible definir a los colombianos sin tener en cuenta los valores, la cultura y las tradiciones de ancestro africano.

Desde esta óptica, mientras la educación propia afrocolombiana, que entre otras cosas debe abarcar su historia, sus conocimientos y técnicas, sus sistemas de valores, sus formas lingüísticas y dialectales, se aplica en los territorios ancestrales, la Cátedra será objeto de implementación en todos los establecimientos públicos y privados del país de acuerdo con lo establecido en el decreto १२2 de 1998. Por lo tanto, el espíritu de la cátedra de estudios afrocolombianos es contribuir, desde un enfoque intercultural, en la transformación de la educación oficial colombiana garantizando la presencia de la diversidad epistémica en el currículo general del país; entre tanto, la educación propia afro hace apuestas por la autonomía territorial, la descolonización mental de los maestros, la recuperación y fomento de la solidaridad, la validación de sus construcciones epistemológicas y el fortalecimiento organizacional y político de las comunidades.

Sin embargo, es preciso aclarar que ni el concepto de educación propia, ni la Cátedra de estudios afrocolombianos son realmente parte integral del currículo nacional. Pese al artículo 68 constitucional que señala la educación para los grupos étnicos como aquella que desarrollará su identidad cultural, el estado omite incluir en el currículo nacional la diversidad de sistemas de pensamiento que dieron origen a la identidad colombiana. Tanto el currículo (art. 76) como el plan de estudios (art. 79) están definidos en la ley 115 de 1994 de manera abstracta y supuestamente neutral, invisibilizando los destinatarios y al mismo tiempo constructores de la cultura nacional. Esta es justamente la reclamación que hace Manuel Zapata Olivella (1990) en su obra autobiográfica Levantate Mulato. La cultura nacional según este autor está integrada por todas las culturas populares del país. El Estado ha definido la presencia de la comunidad negra en el sistema de enseñanza por fuera de la estructura curricular general, razón por la cual, los maestros no asumen el desarrollo de la ley 70 de 1993 y el decreto 1122 de 1998 como algo obligatorio sino discrecional. La institucionalidad educativa en Colombia no considera los aportes de las culturas de origen africano como parte de la identidad nacional, al contrario, la develación de las epistemologías de la diáspora africana las perciben como un espacio concedido por el sistema público de educación a las comunidades negras y se considera un agregado que algunos maestros e instituciones 
educativas consideran innecesario e irrelevante, de ahí la caracterización de la etnoeducación como un servicio que el Estado ofrece (ley १15, titulo III, cap. III).

Por otra parte, el capítulo VI de la ley 70 en su artículo 42 crea la Comisión pedagógica nacional de las comunidades negras. Dicha comisión tiene el encargo de asesorar al Ministerio de Educación en la construcción de la política pública de educación para las comunidades afrocolombianas. La comisión conformada por delegados de todos los territorios negros (Pacifico, Atlántico, Valles interandinos, San Andrés, Antioquia y otros territorios) funciona desde el año 1996 pero su papel misional (asesorar al MEN) está diluido por una suerte de "oficialismo mendigo" ostentado por algunos delegados y cuya articulación con las hipócritas estrategias del Estado procuran desvanecer los debates de fondo.

Por iniciativa de algunos delegados a la Comisión Pedagógica y por mandato de la ley, el Ministerio de Educación dio apertura en 2002 al debate sobre la construcción de la Política Pública Nacional educativa para comunidades afrocolombianas como un documento que consolida, integra y desarrolla la normatividad especial existente. No obstante, la preparación del documento sufrió del mal eterno de las discusiones bizantinas. Mi propia experiencia como coordinador de la Comisión pedagógica nacional (años atrás) me dice que el interés del Estado para la aprobación de un documento que registre estrategias, acciones, estructura administrativa y presupuesto para el desarrollo de la educación propia afrocolombiana es casi inexistente. Sin menospreciar la gestión de los pueblos indígenas, es claro que el Estado tiene mayores consideraciones con estos pueblos a la hora de establecer apoyos, recursos y normativas. Sin ahondar en razones de tipo histórico que desbordaría el alcance de este artículo, se podría decir que en la concepción de la sociedad colombiana la etnicidad en términos de particularismo cultural tiene nombre propio: el indigenismo.

Siguiendo este razonamiento, es fácil comprender que las universidades colombianas que tienen programas de etnoeducacion presenten estructuras académicas inclinadas a promover las cosmovisiones indígenas como si el pensamiento Afro no fuera parte de la historia de Colombia y de América.

En los aspectos claves del derecho a la educación propia, la constitución colombiana de 1991 no hace excepción de un grupo étnico específico. El discurso constitucional habla de derechos para grupos étnicos en general; tal es el caso de lo enunciado en el artículo 68 de la carta política que reza lo siguiente: los integrantes de los grupos étnicos tendrán derecho a una educación que respete y desarrollo su identidad cultural. Sin embargo, la reglamentación de este articulo por la vía del capítulo III de la ley 115 y el decreto reglamentario 804 de 1995 es tomada por el Ministerio de Educación, así como por parte de las universidades solo como derechos de los pueblos indígenas. Las condiciones excepcionales que plantea este decreto para la vinculación de nuevos maestros no son aplicadas en los territorios de las 
comunidades negras. Asimismo no se respetan las autoridades territoriales propias definidas en el mismo decreto para concertar con el Estado.

La identidad de los negros se folcloriza, mientras que las identidades indígenas se respetan. Desde el punto de vista de los estudios subalternos sur asiáticos, las comunidades Negras están aquí en el campo de la violencia epistémica (SPIVAK, 2003), esto es, la negación de su capacidad de pensar y producir conocimientos; así mismo, en el ámbito del pensamiento de Fanon (2009, p. 42) estarían en el estado del No Ser bajo la línea de lo humano, cuya jerarquía la marca con mucha precisión la sociedad colonial de larga duración. De esta manera, se hace evidente que las luchas de los negros en Colombia se complejizan en la medida en que se naturaliza la idea de que esta población está desprovista de derechos más allá de la ciudadanía. Condición ciudadana que tampoco está asegurada del todo y al contrario se encuentra diluida en el doble juego del multiculturalismo constitucional: aceptación-negación.

Todo ello, sin reparar en que el proyecto republicano que construye, desde १८२०, una visión de educación para la democracia no contempló de ninguna forma las poblaciones de origen africano que, pese a su participación activa en las guerras independentistas, fueron llevadas hasta 1851 como sociedades esclavizadas.

A pesar de la existencia de la ley 70 de 1993 y sus concepciones de educación propia afrocolombiana, el Ministerio de educación promueve en los territorios de estos pueblos la estandarización curricular como si las particularidades culturales no fueran patentes. Los anuncios sobre las competencias básicas, laborales y ciudadanas que son parte de la política estatal son mayores que la difusión de una educación endógena Afro (GARCÍA, 2016) que como derecho colectivo está consagrado no solo en decretos y leyes colombianas, sino también en normativas del orden internacional como el convenio 169 de la 0IT, del cual la Corte Constitucional colombiana ha dicho que también tiene como destinatarios a los pueblos negros del pais. ${ }^{3}$

\section{LOS AUANCES DE LA EDUCACIÓN AFROCOLOMBIANA}

Siguiendo los postulados de los intelectuales negros que durante casi todo el siglo XX se pronunciaron en forma vehemente en defensa de la educación para las comunidades afrocolombianas, ${ }^{4}$ algunos dirigentes de los territorios y maestros convencidos de la necesidad de vincular cultura y educación fueron creando desde la década de los ochenta una suerte

Sentencia C-169 de 2001.

Ver mi libro "Por fuera de la casa del amo", Universidad de Nariño-2016. 
de movimiento pedagógico afrocolombiano que se pudo expresar en la puesta en marcha de varias experiencias educativas endógenas diseminadas en distintos lugares del país.

Dos de las más representativas experiencias de educación afro, se dedicaron a la recuperación de las lenguas vernáculas. Efectivamente, San Basilio de Palenque, la tierra del gran cimarrón Benkos Biohó, considerado el primer territorio libre en América, desarrolló un proyecto de educación comunitaria para la reafirmación cultural con base en la defensa y promoción de la lengua palenquera. Lo mismo, ocurrió en San Andrés Islas. Los raizales negros construyeron y aplicaron una propuesta de educación en la línea de retener el habla criolla que por la influencia del español del centro del país venia en franca decaída. El creol, como lo llaman los sanandresanos, es lengua materna y principio para sostener las prácticas ancestrales de los territorios insulares.

En los valles interandinos (norte de departamento del Cauca y sur del Valle) en los años ochenta florecieron varias experiencias educativas propias. Algunas de ellas son: "Casita de niños", este proyecto de carácter comunitario es una red de preescolar que se crea para la promoción de la educación inicial en tiempos de un vacío normativo oficial. Inician desde 1979 en Villarrica-Cauca, en 1980 en Quinamayó municipio de Jamundí-Valle, en 1984 en la Balsa, municipio de Suarez Cauca y en 1985 en San Nicolás municipio de Caloto. Su objetivo es el desarrollo de la identidad cultural de las comunidades negras dentro del marco de la interculturalidad critica, ${ }^{5}$ potenciando la creatividad, el sentido crítico y el desarrollo de las habilidades y destrezas tanto individuales como colectivas, necesarias para el desarrollo y transformación del medio (LARRAHONDO, 2004; MINISTERIO DE EDUCACIÓN NACIONAL, 2004). Esta experiencia educativa es particularmente importante en tanto se percibe como reacción de las comunidades negras frente al proyecto industrial de la caña de azúcar que, desde las primeras décadas del siglo XX, les arrebató sus tierras y desarticuló sus prácticas culturales.

Por otro lado, la Experiencia educativa comunitaria Villa Paz-Jamundi-Valle, proyecto ubicado en el poblado de Villapaz en el municipio de Jamundí -Valle del Cauca fue iniciado en 1984. Colegio de educación media vocacional, con modalidad agrícola y con énfasis comunitario. Es una institución que parte de las características de la comunidad, pensada por ellos mismos, quienes se definieron como autogestionarios, comuneros, participativos, solidarios, con fuerte arraigo a la tierra y a sus valores ancestrales (MINISTERIO DE EDUCACIÓN NACIONAL, 1994). El proyecto fue preparado por los jóvenes bachilleres de la época que querían garantizar en su pueblo el paso de la primaria a la secundaria sin que los estudiantes tuvieran que alejarse de la comunidad. No obstante, los fundamentos del proyecto educativo de Villapaz fue resultado del encuentro de los mayores con los jóvenes para fijar los principios que deben regir la escuela. En una frase recogieron el valor autonómico de la propuesta educativa y

El agregado es mio. 
el anhelo de mantener la condición de "comuneros": "Los límites del colegio son los límites de la comunidad."6

Las experiencias descritas son solo una muestra de las iniciativas de maestros y comunidades que han decidido dar impulso a la concepción de Educación Afro desde una perspectiva endógena. Se podrían mencionar también experiencias educativas en Tadó, Quibdó y medio Atrato en el departamento del Chocó, así como en Nariño, la Costa caucana y Buenaventura. Todas estas iniciativas y las que no es posible describir por los límites de este artículo, fueron socializadas en el marco de cinco grandes eventos en que los promotores de la educación propia o etnoeducación afrocolombiana se dieron cita creando verdaderos escenarios de encuentro entre la intelectualidad y el movimiento social. El primero de los eventos se desarrolló en Quibdó-Chocó (ENCUENTRO DE PASTORAL AFROAMERICANA, 1991) con el nombre de Quinto Encuentro de Pastoral Afroamericana. Este encuentro concitó la presencia de líderes negros de varios países de América Latina y con su enfoque de educación liberadora, se constituye en la apertura del debate nacional de los noventa, sobre los derechos de los negros a una educación basada en sus tradiciones culturales y epistemológicas y, por lo tanto, alejada de las directrices estatales. El segundo evento se llevó a cabo en Tumaco-Nariño y fue llamado Primer Congreso nacional de etnoeducacion afrocolombiana en (1992). Mientras el de Quibdó fue convocado por la Pastoral Afrocolombiana, el de Tumaco es el primero que convocan los propios maestros negros en el contexto de discusión nacional sobre el artículo transitorio 55 de la Constitución Política. Los eventos que siguieron Cartagena 1993, Guapi-Cauca 1994 y Chinauta-Cundinamarca 1997 se consideran continuación de los debates nacionales que responden a la pregunta 己̇es posible una educación diferenciada para la población afrocolombiana? 0 ¿̇Cómo el Estado debería asumir la atención educativa en los territorios de las comunidades negras? Los años noventa del siglo XX se pueden considerar como la década de la emergencia y consolidación del movimiento pedagógico afrocolombiano. Sin duda, las discusiones suscitadas en estos encuentros, sumadas a los planteamientos de los intelectuales y las dinámicas político-pedagógicas de las experiencias de educación comunitarias, fueron dando forma a lo que he llamado Pensamiento Educativo Afrocolombiano (2016).

Después de este recorrido por experiencias educativas comunitarias y eventos nacionales de debate y crítica del sistema educativo oficial, el proceso de reivindicación por una educación propia cae en una especie de vacío. Sin embargo, cuando el Ministerio de Educación (2004) anuncia el concurso para proveer cargos de maestros afrocolombianos y expidió el decreto 3323 mediante el cual la entidad oficial organizaría dicho concurso, el movimiento social afrocolombiano, a través de la gestión de la Comisión pedagógica nacional, retoma con fuerza las reivindicaciones en educación. Si no fuera porque ese concurso

6 Por fuera de la casa del amo (p. 153). 
violentaba toda la normatividad favorable a las comunidades negras parecería algo normal y benéfico para estos pueblos.

Esta convocatoria inconsulta y además engañosa del Ministerio de Educación, donde se ponen en concurso público y nacional las plazas de maestros negros ocupadas con varios años de antelación, no solo se percibió como una violación de derechos sino también como un atentado a la estabilidad laboral de los docentes de los territorios Afro. A partir de este debate se revive el movimiento pedagógico nacional de comunidades negras en 2005. Algunos delegados de departamentos a la Comisión Pedagógica se pusieron del lado del Ministerio de Educación, mientras que otros (incluyendo quien escribe) optaron por desobedecer la norma en tanto que se consideraba no ajustada a los derechos educativos afrocolombianos.

El conflicto entre la comunidad afro y el Ministerio por el concurso de maestros aún no se ha resuelto. Sin embargo, el debate ha servido para que las regiones más beligerantes, específicamente la población negra del departamento de Nariño, quienes hicieron desobediencia civil en tres oportunidades la convocatoria de dicho concurso, recibieran apoyo en el año 2009 de parte del propio Ministerio de educación (dato curioso) para la construcción del proyecto de educación propia.

El Ministerio llamó a esta iniciativa Proyecto Etnoeducativo Afronariñense y las comunidades a partir de ahí lo denominan PRETAN. El apoyo sirvió para consultar en catorce municipios con población afro el tipo de educación que se requiere. Más de noventa eventos educativos con amplia participación comunitaria dieron como resultado el documento marco de la educación propia en Nariño. De esta manera, el PRETAN es una guía conceptual, teórica, pedagógica, metodológica y operativa mediante la cual se orientan los procesos educativos en las comunidades afronariñenses (MINISTERIO DE EDUCACIÓN NACIONAL, 2011). En desarrollo de la legislación educativa Afro, este documento contiene el modelo pedagógico conocido como Aprendizaje Colectivo, el cual busca la participación de los miembros de la comunidad en el proceso de aprendizaje de las niñas y niños afro. Desde esta perspectiva, el maestro no es la única fuente del saber. El conocimiento es resultado de la interacción de los miembros de la comunidad en un dialogo permanente. Se postula desde ese modelo que el aprendizaje es una experiencia comunitaria y cultural a contrapelo de lo que plantean otras pedagogías convencionales. El centro del modelo es la comunidad y no un actor social en particular.

En la actualidad, el PRETAN está aprobado en la Asamblea departamental de Nariño (2013) como política pública de educación afronariñense (Ordenanza número 37 de 2013). Bajo criterios de transferencia de competencias y autonomía educativa territorial, dicha política se plantea como objeto reconocer a las comunidades negras del departamento de Nariño el derecho a una educación que valore, respete y desarrolle su identidad cultural 
en los niveles de educación inicial, básica, media y superior de acuerdo con los intereses sociales, culturales y políticos de estas poblaciones.

Este es un hecho significativo dado que en ningún otro territorio del país se ha logrado concretar un acuerdo entre comunidades y el gobierno para la definición de una política educativa especial. Si bien el documento de política pública de Nariño es nuevo ya tiene resonancia en el departamento y el país. Sin embargo, es necesario poner atención en lo siguiente: pese a que ya es un hecho la aprobación oficial de la Política Publica la garantía del desarrollo y aplicación de la misma parece no estar en el terreno de los entes oficiales, ni a nivel nacional, ni a nivel departamental. Lo que realmente ha ocurrido es una institucionalización de los beligerantes procesos de desobediencia al concurso de los maestros Afro en la Costa de Nariño. Varios años después de su promulgación, el documento de Política Pública Afronariñense duerme en los anaqueles y no es más que letra muerta, con lo que se puede inferir que el proyecto de inclusión de los procesos educativos afrocolombianos por parte del Estado no es otra cosa que una estrategia racista de desmovilización de las comunidades desobedientes e insumisas. El multiculturalismo de Estado, en forma premeditada crea la norma para cerrar los procesos reivindicativos, soslayar la construcción de pensamiento y evitar las protestas. El efecto buscado con la normatividad incluyente es la percepción comunitaria que sus derechos hacen parte de la ley y por tanto tendrán solución. Pero, la tranquilidad producto de esa sensación no solo estanca los procesos, también desvanece las condiciones y posibilidades de una nueva critica al Estado.

Por lo tanto, las leyes que reconocen derechos educativos a los negros en Colombia funcionan como una suerte de catarsis para el adormecimiento social, bálsamo para los dirigentes comunitarios que han cesado en la reclamación de este derecho y quizás se presenta también como una re-elaboración del duelo histórico de la esclavización. Con todo, aún es posible excepcionar de este marasmo colectivo a un grupo de docentes-líderes de la Costa de Nariño que insisten incansablemente en sus luchas por hacer respetar sus derechos como maestros negros. El logro de la sentencia C- 666 de 2016 expedida por la Corte Constitucional donde se ordena al Congreso la creación de un estatuto especial de educación para los pueblos negros, es fiel testimonio de la insumisión de este grupo de maestros afronariñenses. Para llegar a este punto, el movimiento profesoral negro de Nariño desarrolló actividades de protesta fuertes durante diez años, entre ellas, en dos ocasiones, la toma de la iglesia de San Francisco en pleno centro histórico de Bogotá.

Por otra parte, a escala nacional el Ministerio de Educación en varios momentos ha intentado institucionalizar las experiencias educativas de las comunidades Afro etiquetándolas como proyectos etnoeducativos. En Cartagena (1993) y Guapi (1994) bajo la figura de Seminarios- Talleres nacionales de Etnoeducación Afrocolombiana, arrancan los procesos de asimilación de las iniciativas educativas de la comunidad Afro, ante todo rurales, 
incorporándolas a la definición oficial según la cual la etnoeducación es un servicio del Estado (ley 115/94, cap. III). Presentada de esta forma, la etnoeducación pierde su valor político y su fuerza argumentativa como proceso emancipatorio. Es justo por estas razones que me he desprendido del concepto de etnoeducación, dada su afiliación a las políticas generales del Estado y a cambio he promovido la idea de trabajar en los territorios negros desde la concepción de Educación propia afrocolombiana. Por lo tanto, la etnoeducación en términos de educación propia Afro comporta una historia diferente, contiene un proyecto de liberación y pretende una descolonización de la pedagogía, un fortalecimiento en la capacidad organizativa de las comunidades y la autonomía de los territorios.

Ahora bien, en materia de construcción de Política Pública Nacional de educación Afro, las discusiones entre el Ministerio de Educación y la Comisión Pedagógica de las comunidades negras avanzan hacia la consolidación de un documento llamado Política de Educación Intercultural Afrocolombiana. Mientras que a otros pueblos se les permite hablar de educación propia, a las comunidades afro el Estado le asigna el concepto aparentemente despolitizado de interculturalidad como una forma de flexibilizar y sobre todo aligerar la carga semántica que está detrás de los conceptos de educación Afro o educación propia. Se deja ver aquí la estrategia del multiculturalismo en el sentido de crear toda una eufemística perversa para dislocar el discurso emancipador de los pueblos de la diáspora africana en América. El concepto de interculturalidad promovido desde el Estado contiene una trampa lingüística. Se ofrece como escenario de encuentro, comunicación y convivencia entre las etnias y culturas del país pero en el fondo solo responde a una estrategia de la política de inclusión multicultural. Por lo tanto, para la democracia liberal, sistema de gobierno en la mayor parte de los países de América, interculturalidad es sinónimo de multiculturalismo de Estado.

Ahora bien, el aporte de los intelectuales negros del siglo XX y las experiencias de educación propia afrocolombiana han generado suficientes elementos de tipo epistémico, pedagógico y conceptual para la formulación de una política pública nacional integral desde la perspectiva de movimiento social..$^{7}$ Algunos de estos elementos se pueden resumir de la siguiente manera: a) la educación afro, en términos de educación propia, es un proyecto de tipo histórico y como tal parte de una crítica fuerte al capitalismo y a sus políticas de violencia y despojo contra los pueblos y territorios; b) la educación afro es un proyecto endógeno, es decir, para que en ejercicio de la autonomía las comunidades decidan el perfil de su proyecto educativo y el destino de sus pueblos tomando como base sus necesidades e intereses sociales, culturales, ambientales y políticos; c) la educación propia afrocolombiana es un proyecto de tipo cultural en la medida en que se plantea el rescate y valoración de sus tradiciones y construcciones sociales en general. La Cultura Negra, como la llamaran los pensadores afrocolombianos del siglo XX, supera la idea de las danzas y los ritmos

Lo que sigue en este apartado es tomado de mi libro "Por fuera de la casa del amo" (GARCÍA, 2016). 
musicales autóctonos que han sido tomados como únicos elementos de la identidad en los pueblos negros. Por el contrario, en el concepto de Cultura Negra, de acuerdo con Córdoba (1980, p. 21), se puede percibir como una construcción política que busca la unidad de los afrocolombianos en una forma de contraparte a la dispersión reinante; y por otro lado, el maestro Olivella (2004, p. 134) ha escrito que la Cultura Negra está asociada a las primigenias construcciones sociales del ser humano. En concreto, plantea que las principales contribuciones de nuestras comunidades fueron tres elementos fundamentales: el aporte de haber gestado por vez primera expresiones humanas, el aporte de haber creado por vez primera el núcleo de la familia y el aporte de haber creado las artes, la filosofía, la religión, el concepto universal de lo que es el hombre, como ente creador de valores espirituales y materiales; d) La educación afro es un proyecto pedagógico en el sentido de un reto para los maestros afro pero también para el país; ya que como lo plantea Mosquera (1999, p. 37), es necesario cambiar de actitud y reflexionar sobre el enorme daño psicológico que se ha causado a los estudiantes y comunidades al propiciar la reproducción del etnocentrismo "blanco" y el racismo en la práctica pedagógica cotidiana; finalmente, la educación propia afrocolombiana se concibe también como un proyecto epistémico en el sentido de su propósito de formar los intelectuales que defenderán los derechos de los pueblos negros colombianos; es decir, las nuevas generaciones de intelectuales negros que saldrán de estos procesos garantizaran la continuidad de las luchas y reivindicaciones afrocolombianas, como lo hicieran los pensadores negros del siglo XX ya mencionados, y como quedó demostrado en las experiencias educativas de iniciativa comunitaria en las décadas de los años ochenta y noventa del mismo siglo. Generar la intelectualidad que discutirá con el Estado los derechos de los pueblos negros de Colombia será el papel principal de la educación en perspectiva afrocolombiana.

\section{EN CONCLUSIÓN}

La lucha por una educación que respete y desarrolle la identidad cultural y la subjetividad colectiva de las poblaciones afrocolombianas, ha llevado a sus dirigentes, intelectuales, maestros y líderes comunitarios por caminos sinuosos y tortuosos durante todo el siglo XX y hasta la actualidad. La negativa del Estado a reconocer los derechos de estos pueblos en sentido práctico es un proceso que sigue su curso, mientras que las comunidades echan mano, casi que inútilmente, de sus acumulados históricos y de las leyes colombianas de derecho etnico para establecer puentes con la institucionalidad y fortalecerse internamente para mantener el debate. 
Los intelectuales negros durante todo el siglo XX dieron pistas, cada uno desde su campo de acción, para que la población afro se organizara frente al Estado en la reclamación de sus derechos educativos. El movimiento social asumió el pensamiento de los intelectuales (pensamiento diasporico) y lograron crear escenarios de debate con los gobiernos durante las dos últimas décadas, construyendo experiencia de educación propia en franca desobediencia a los mandatos oficiales. Los proyectos educativos mencionados aún persisten pese a las estrategias tecnócratas y domesticadoras de la educación oficial que sigue reduciendo la educación de los pueblos negros al campo disciplinar sin articular el aprendizaje a los contextos culturales y sin valorar las visiones epistemológicas de estas comunidades.

Los derechos a la educación afro negados sistemática e históricamente por el alto gobierno solo empiezan a concretarse en el departamento de Nariño mediante la ordenanza 037 de 2013 con la cual se aprueba la política pública de educación para comunidades negras de este territorio. Se espera que Nariño se constituya en ejemplo para las demás regiones del país que tienen importantes porcentajes de población con ancestro africano. Sin embargo, esa política, financiada por el Ministerio de educación y aprobada por la Asamblea departamental de Nariño no tiene ninguna aplicación práctica, no cuenta con asignación presupuestal y todo indica que fue resultado de una estrategia premeditada de "inclusión" para desactivar los procesos que desobedecen las reglas del Estado reclamando atención educativa diferencial.

En evento reciente celebrado en Tumaco-Nariño (julio de 2018), las organizaciones mayores de comunidades negras, sindicatos y consejos comunitarios confrontaron de nuevo con funcionarios del Ministerio de educación y las autoridades educativas departamentales en temas de procedimientos legales para la vinculación de nuevos maestros. Por el ambiente percibido en estas jornadas de trabajo, se puede presagiar que la puesta en marcha del estatuto especial de profesionalización docente para las comunidades negras, lactualmente pendiente de aprobación en el Congreso nacional), podría reactivar con mucha fuerza el movimiento pedagógico afrocolombiano.

\section{REFERENCIAS}

ASAMBLEA DEPARTAMENTAL DE NARIÑO. Política pública de educación Afronariñense. Pasto: Archivo de la Asamblea, 2013.

CAICEDO ORTIZ, J. A. A mano alzada... Memoria escrita de la diáspora intelectual afrocolombiana. Cali: Sentipensar Editores, 2013.

CÓRDOBA, A. Cultura negra y avasallamiento cultural. Bogotá: MAP Publicaciones, 1980.

ENCUENTRO DE PASTORAL AFROAMERICANA, 5., 1991, Quibdó. Memorias y Conclusiones. Quibdó: Diócesis de Quibdó, 1991. 
FANON, F. Piel negra mascaras blancas. Madrid: Ediciones Akal, 2009.

GARCÍA, J. Etnoeducación Afro "Casa Adentro". Revista Pedagogía y Saberes, Bogotá: Universidad Pedagógica Nacional, 2011.

GARCÍA, J. La tradición oral, una herramienta para la etnoeducación. Quito: Génesis Ediciones, 2001.

GARCIA, J. Por fuera de la casa del amo: insumisión epistémica o cimarronismo intelectual en el pensamiento educativo afrocolombiano siglo XX. Pasto: Universidad de Nariño, 2016.

GROSFOGUEL R.; CASTRO-GÓMEZ, S. El Giro decolonial. Relexiones para una diversidad epistémica más allá del capitalismo global. Bogotá: Siglo del Hombre Editores, 2007.

HALL, S. Da Diaspora: identidades y mediaciones culturales. Belo Horizonte: Ed. UFMG, 2006.

LARRAHONDO, S. En Memorias: Primer Foro Nacional de Etnoeducación Afrocolombiana. Bogotá: Ministerio de Educación, 2004.

MAYA, A. Atlas de las Culturas Afrocolombianas. Bogotá: Ministerio de Educación, 2003.

MAYA, A. Demografía histórica de la trata por Cartagena 1533-1810. En: Geografía Humana de Colombia, Los Afrocolombianos. Bogotá: Instituto Colombiano de Cultura Hispánica, 1998.

MAYA, A. Diasporas africanas en Colombia. Visibilidad e invisibilización de los legados de las culturas de África occidental, en tiempos del bicentenario de la independencia. En: CANTOR, R. B. (ed.). Rutas de libertad, quinientos años de travesía. Bogotá: Ministerio de Cultura-Pontificia Universidad Javeriana, 2010.

MINISTERIO DE EDUCACIÓN NACIONAL. Asocoetnar. Proyecto Etnoeducativo Afronariñense. Tumaco: [s.n.], 2011.

MINISTERIO DE EDUCACIÓN NACIONAL. Primer Foro nacional de Etnoeducación Afrocolombiana. Memorias. Bogotá: [s.n.], 2004.

MINISTERIO DE EDUCACIÓN NACIONAL. Primer seminario taller de etnoeducacion para comunidades afrocolombianas. Memorias. Bogotá: [s.n.], 1994.

MOSQUERA, J. La etnoeducacion afrocolombiana: guía para maestros, líderes y comunidades. Bogotá: Editorial Magisterio, 1999.

LAO-MONTES, A. Hilos descoloniales. Trans-localizando los espacios de la diáspora africana. Tabula Rasa, v. 7, p. 47-79, 2007.

OLIVELLA, Z. M. Changó el gran putas. Bogotá: Rei Andes, 1992. 
OLIVELLA, Z. M. iLevántate Mulato!! por mi raza hablará el espiritu. Bogotá: Rei-Andes, 1990.

OLIVELLA, Z. M. MEN - Memorias I Foro nacional de etnoeducacion afrocolombiana. [S.l.]: [s.n.], 2004.

QUIJANO, A. Colonialidad del poder, eurocentrismo y América latina. Lima: Centro de Investigaciones Sociales, 2000.

SPIVAK, G. ¿̇Puede hablar el Subalterno? Revista Colombiana de Antropología, Bogotá, v. 39, 2003.

WALSH. Interculturalidad, colonialidad y educación (ponencia en Primer seminario internacional Etnoeducación, multiculturalismo e interculturalidad. Bogotá: [s.n.], 2005.

Enderezos para correpondencia: Carrera 46 \# 51-58, Barrio Ciudad Córdoba, Cali, Colombia; Jegar2013@hotmail.com 
